\title{
Implementing Schema-theoretic Models of Animal Behavior in Robotic Systems
}

\author{
Khaled S. Ali and Ronald C. Arkin \\ Mobile Robot Laboratory \\ College of Computing \\ Georgia Institute of Technology \\ Atlanta, GA, 30332-0281 USA \\ $\{k a l i, a r k i n\} @ c c . g a t e c h . e d u$
}

\begin{abstract}
Formal models of animal sensorimotor behavior can provide effective methods for generating robotic intelligence. In this paper we describe how schema-theoretic models of the praying mantis are implemented on a hexapod robot equipped with a real-time color vision system. The model upon which the implementation is based was developed by ethologists studying mantids. This implementation incorporates a wide range of behaviors, including obstacle avoidance, prey acquisition, predator avoidance, mating, and chantlitaxia behaviors.
\end{abstract}

\section{Introduction}

Ecological robotics refers to incorporating aspects of the relationship a robot maintains with its environment into its control system (i.e., its ecology) [4]. One means for developing such a control system is by exploiting models of behavior developed by ethologists or neuroscientists. Although considerable research has been conducted in the modeling of neural controllers based on animal models (e.g., [3, 5, 14]), incorporation of environmental interactions has been far less studied within the robotics community. Although some work has been undertaken within the artificial life arena $[10,11]$, almost all of this work has been conducted in simulation or at best on primitive robotic implementations.

In this paper we expand upon our earlier simulation studies reported in [4] and report results obtained on the implementation of a model of praying mantis behavior on a robotic hexapod equipped with a real-time vision system. As we are working with models generated by animal scientists we hope that not only will these results have value within the robotics community in terms of providing a path for generating intelligent behavior in machines, but that they may also serve as a basis for feedback for stimulation, regeneration, and refinement of the animal models themselves.

\section{Schema-theoretic Approach of Pray- ing Mantis Behavior}

Schema theory is a powerful and expressive means for describing behavior, both neuroscientific [1] and robotic [2, 12]. Schemas themselves are distributed concurrent processes, charged with enacting the internal behavioral goals and intentions of the agent in response to external stimuli. The output of these processes can be combined in a host of ways including, for example, priority-based arbitration (subsumption) [6], behavioral fusion [2], and action-selection [13] to name a few.

Our research has focussed on ethological models of visuomotor behavior for the praying mantis. An ethogram for this creature appears in Figure 1 [4]. It encompasses a wide range of behaviors ranging from exploration, locomotion, rest, eating, mating, and defensive activities. From this model, we have implemented a subset of these behaviors including simple locomotion incorporating obstacle avoidance, prey acquisition (for eating), predator avoidance (for survival), mating, and chantlitaxia behavior [8], where the agent searches for a hospitable environment, which in the case of the mantis is low brush or bushes when young and trees when older.

Our current model also incorporates motivational variables (shown in parentheses) which affect the selection of motivated behaviors such as predator avoidance (fear), prey acquisition (hunger) and mating (sex- 


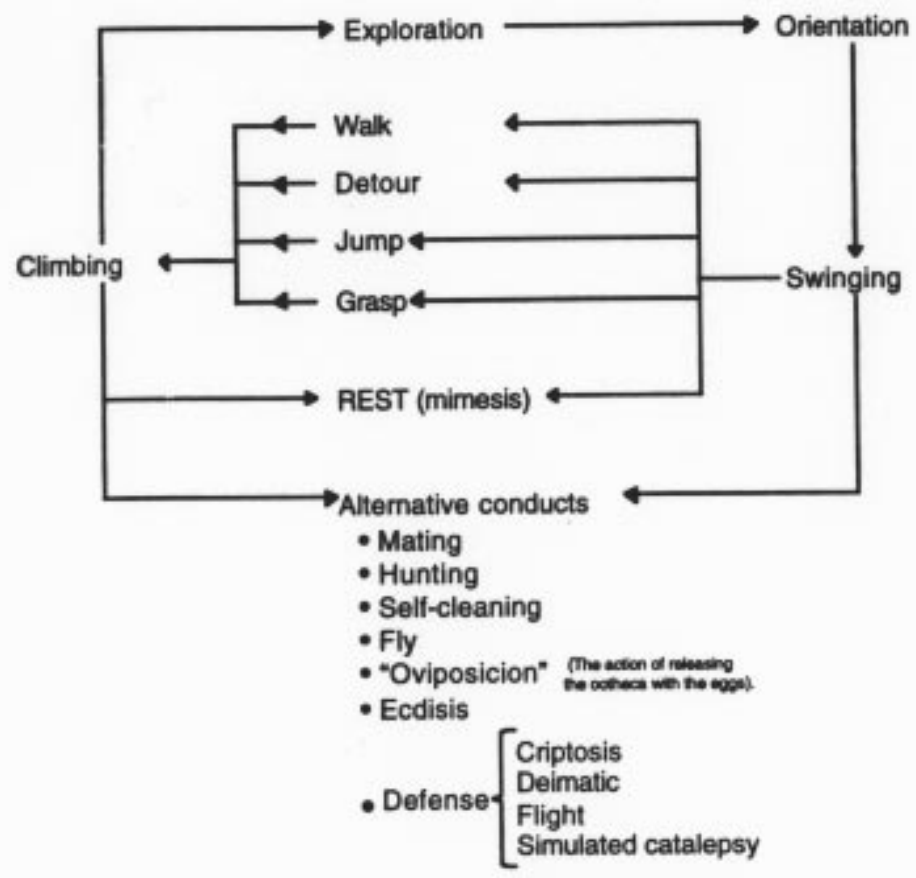

Figure 1: Ethogram of praying mantis behavior. (From [4])

drive). These variables are currently modeled quite simply (described in Section 3) but are intended to be extended to incorporate factors such as diurnal, seasonal, and climatic cycles and age-related factors. This simplified model, depicted in Figure 2, serves as the basis for the implementation described in this paper. It is derived from a model of praying mantis behavior developed by Cervantes-Pérez [7]. (Figure 3). This model makes a weak commitment to an action-selection mechanism (modeled as lateral inhibition in Fig. 3) for arbitration between prey acquisition, predator avoidance, and mating using a colonystyle architectural strategy [9] for arbitration between the winner of the motivated behaviors, obstacle avoidance, and chantlitaxia. The outputs of these behaviors are encoded in the form of two percentages, one representing the forward motion as a percentage of the maximum forward speed, and the other representing the turning motion as a percentage of the maximum turning speed (with negative percentages representing turns in the other direction). In the implemented version described below, vision provides the stimuli for chantlitaxia and the set of motivated behaviors while obstacle avoidance is triggered by the hexapod's whiskers.

\section{Robotic Implementation}

This model of praying mantis behavior has been implemented on our robot Miguel. Miguel is a Hermes II hexapod robot manufactured by IS Robotics. It has a color camera mounted at its front, feeding live video into a Newton Research Labs Cognachrome Vision System. Additionally, Miguel has two whiskers mounted at its front that serve as contact sensors, infrared proximity sensors on each leg, and contact sensors on its underside.

The portion of Cervantes-Pérez's model that corresponds to moving-objects as shown on the left side of Figure 2 is implemented on the Cognachrome Vision processor. In our implementation, however, rather than responding to movement, the system responds instead to colors. Green objects represent predators, purple objects represent mates, orange objects that are at least twice as tall as they are wide represent hiding-places, and all other orange objects represent prey. Figure 4 shows the algorithm running on the vision processor. The robot maintains three internal variables that represent the robot's hunger, fear, and sex-drive. Initially, the values of each of these variables is zero. The hunger and sex-drive levels increase 


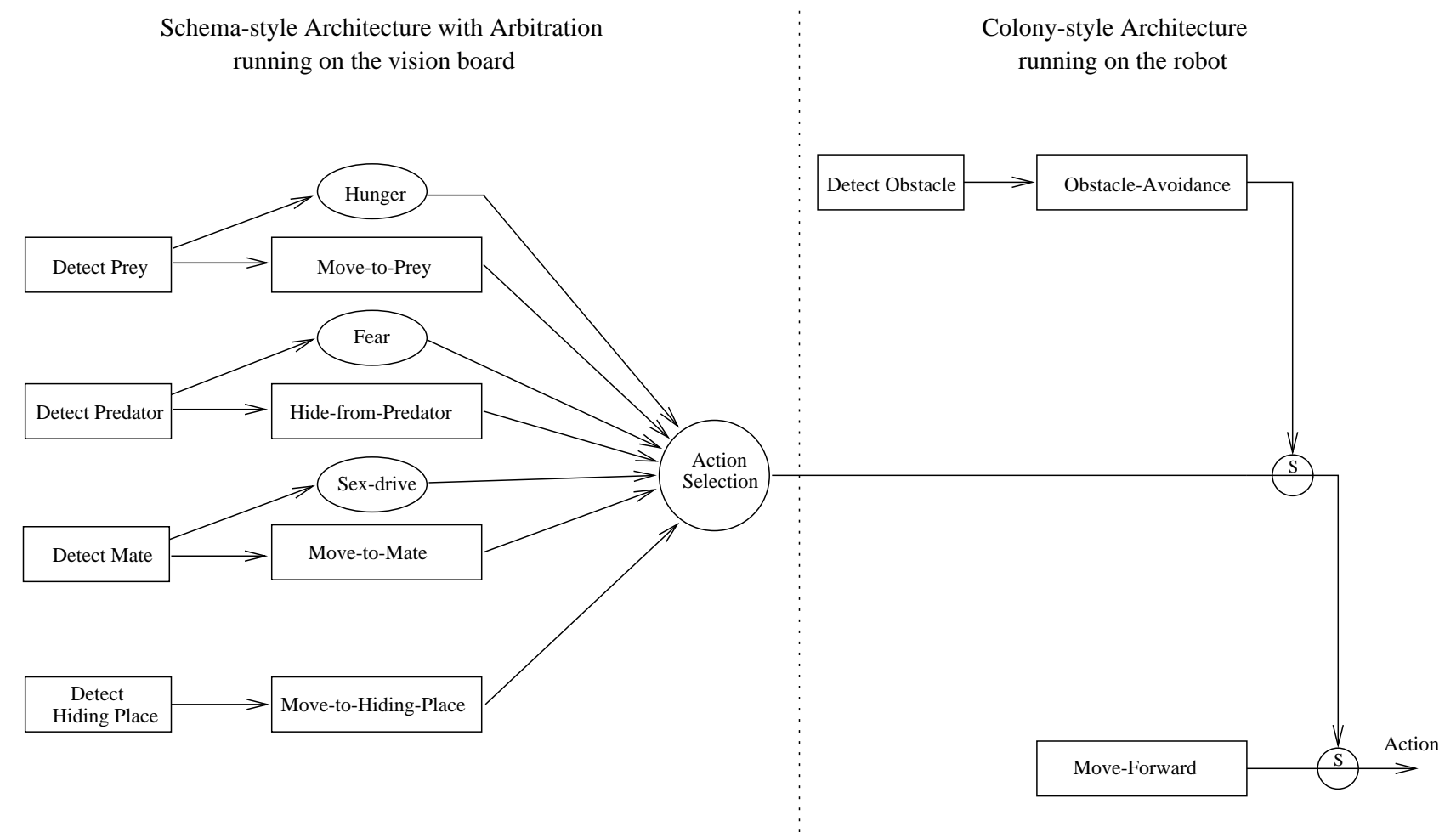

Figure 2: The model implemented on Miguel. It incorporates aspects of action-selection arbitration and colony-style architecture suppression. The interactions of the modules is based on a model of the praying mantis's behavior developed by Cervantes-Pérez [7].

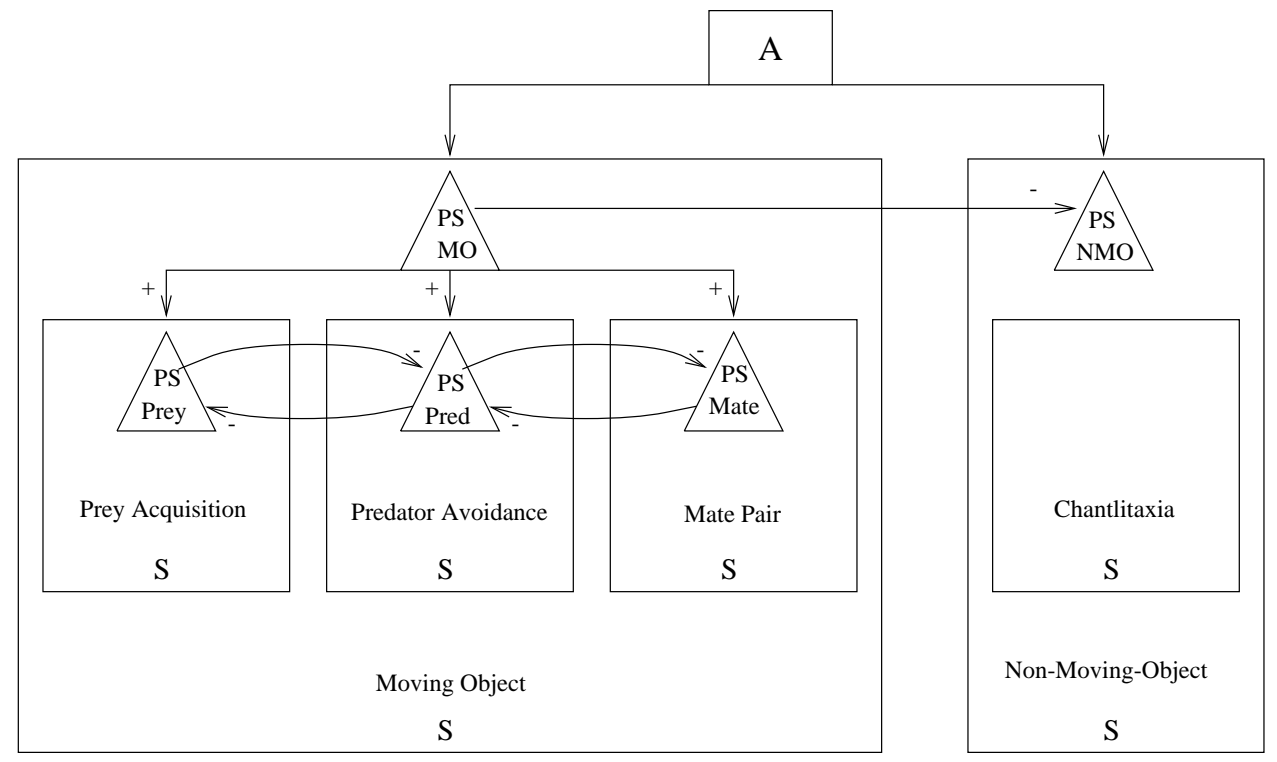

Figure 3: Model of praying mantis behavior developed by Cervantes-Pérez [7]. 
linearly with time, with the hunger increasing at twice the rate as the sex-drive. When the robot has contacted a prey or mate, the robot is considered to have eaten or mated with the object, and the relevant variable resets to zero. Contact is determined by the position of the prey or mate blob in the image captured by the camera on the front of the robot. In this case, the object is considered to be contacted when the bottom of the object blob is in the lower five percent of the image. The fear level remains zero until a predator becomes visible. At that time, the fear variable is set to a predetermined high value. When the predator is no longer visible, the fear level resets to zero. It is possible to incorporate more complex modeling, including habituation to stimuli, but that remains for future work.

The move-to-prey behavior produces a direction that will move the robot toward the largest visible prey, based on the input from the Detect-Prey perceptual schema. Similarly, the move-to-mate and move-to-hiding-place behaviors output directions that will move the robot towards the largest mate and the largest hiding-place, respectively, based on the input from their corresponding perceptual schemas. The hide-from-predator behavior outputs a Stop command if the Detect-Predator schema indicates that there is a predator visible and outputs DONT-CARE otherwise. The output of these behaviors are discrete directions or commands of the following nature: Right, Left, Forward, Forward Right, Forward Left, Backward, and Stop.

The values of the three internal variables (hunger, sex-drive, and fear) and the currently visible stimuli (prey, mates, predators, and hiding-places) are used by the action selection module to select the appropriate action to send to the robot's processor. The motivational variable with the greatest current value is chosen. If there is an associated stimulus present, such as a prey for the hunger variable, then the output of the corresponding behavior is sent to the robot. If there is no associated stimulus visible, then this process is repeated with the motivational variable with the next greatest value. If there is no associated stimulus visible for any of the three motivational variables, but there is a hiding-place visible, then the output of the move-to-hiding-place behavior is sent to the robot. Otherwise, if there are no predators, prey, mates, or hiding-places visible, then the action selection mechanism does not send any command to the robot. There is no predetermined hierarchy or layering; the action chosen depends directly upon the value of the motivational variables and visible stimuli at that moment in time.

For example, if the current values of the motivational variables hunger, sex-drive, and fear are 568, 343 , and 0 , respectively, and there are a prey, mate, and hiding-place visible in the environment, then the action selection module will send the output of the move-to-prey behavior to the robot. This is because the hunger variable has the greatest value and a prey is visible. If the motivational variables are the same as above, but only a mate and hiding-place are visible in the environment, then the output of the moveto-mate behavior will be sent, since the sex-drive variable has the greatest value among the motivations with currently visible stimuli associated with them.

The remaining part of the model, as shown on the right side of Figure 2, is a colony-style architecture [9] with three levels. This part of the model runs entirely on the processor in the Hermes II. Each level contributes in determining the overall behavior of the robotic agent. The output of higher-level behaviors can override the output from a lower-level behavior. Effectively, this causes the output of the higher-level behavior to replace the output of the lower-level behavior for a predefined amount of time.

At the lowest level is the move-forward behavior. This behavior directs the robot to move forward in search of some stimuli. If there is a stimulus such as a predator, prey, mate, or hiding-place visible in the environment, then the action selection module on the left side of Figure 2 will produce an output. This output is translated from a direction or Stop command to spin and speed commands that the robot's builtin Walk behavior understands. These commands will subsume the output of the move-forward behavior for one second. However, if the stimulus is still visible after the one second, the action selection module will continue to produce an output, and this output will repeatedly subsume the lower-level outputs. Finally, the obstacle-avoidance behavior causes the robot to back up a few steps and then turn to the side when an obstacle is detected by a contact with one of the whiskers. When the obstacle-avoidance behavior generates commands, it suppresses the output from the lower behaviors for one second.

The model shown in Figure 2 was implemented incrementally. First, the move-forward behavior was created. Then the obstacle-avoidance behavior was added. Next, prey tracking behavior was developed. When this was working properly, the predator response, and later the mate tracking and hiding-place tracking behaviors were added. 
1. Increment sex-drive and hunger, and set fear.

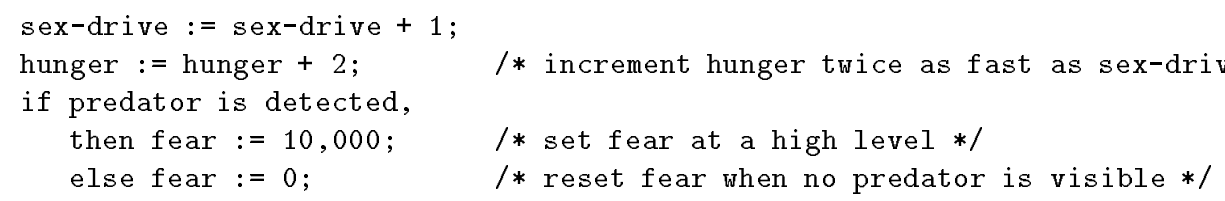

2. Check if mate or prey are close enough to eat.

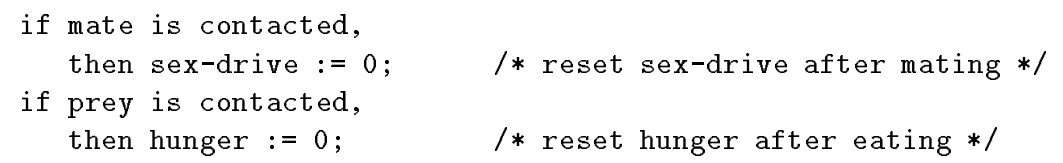

3. Each behavior produces a direction or Stop command, based on the input from its corresponding perceptual schema.

(a) move-to-prey, move-to-mate, and move-to-hiding-place

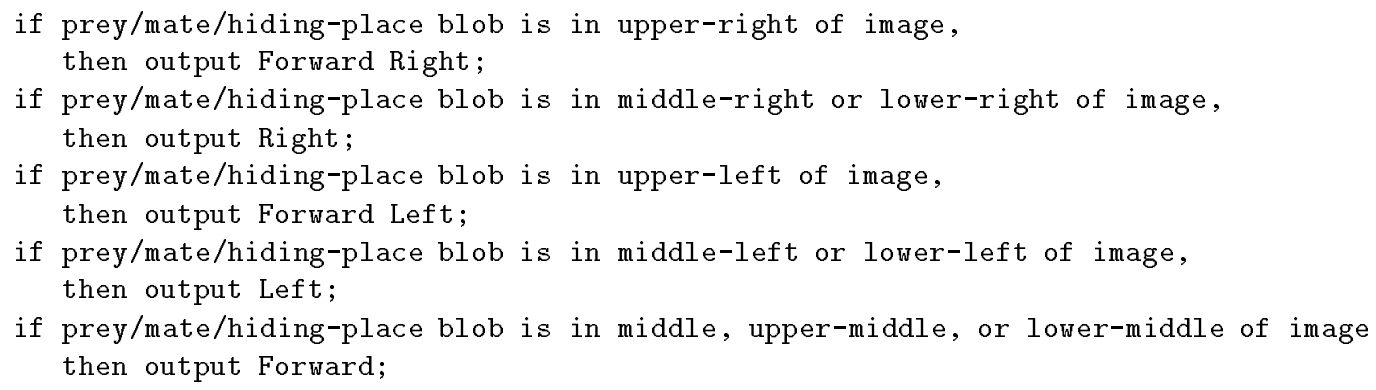

(b) hide-from-predator

if predator is detected, then output Stop, else output DONT-CARE;

4. Choose an output from a behavior, to pass along to the robot.

if there is an associated stimulus for the motivational variable with greatest value, then output direction from behavior corresponding to this variable, else if there is an associated stimulus for the motivational variable with second greatest value, then output direction from behavior corresponding to this variable,

else if there is an associated stimulus for the motivational variable with third greatest value, then output direction from behavior corresponding to this variable, else if there is a hiding-place visible,

then output direction from $\{\backslash b f$ move-to-hiding-place behavior $\}$ else do nothing;

Figure 4: Algorithm associated with the schema-style architecture running on the vision processor. 


\section{Robotic Results}

To test the performance of the implemented model, Miguel wanders around our lab, responding to colored boxes used to represent a predator, prey, mate, and hiding-place. When the execution begins, the hunger, fear, and sex-drive levels are all zero. Therefore, if a predator is detected, Miguel freezes, regardless of whether there is prey or mate visible. Furthermore, if Miguel sees both prey and mate, but no predator, it will move toward the prey, since the hunger increases faster than the sex-drive. If Miguel has not eaten for a long time, then its hunger level will increase beyond the static level that fear is set at when a predator is visible. In this case, if both predator and prey are visible, the robot will move toward the prey even though there is a predator in sight. This also is true when the robot has not mated for a long time.

The following is a description of one particular execution sequence, shown in Figure 5. When the robot was started, the predator, prey, and mate were all in view. In the first picture, Miguel is remaining motionless, because there is a green box representing a predator in his view just out of the picture to the right. Then, the predator was removed from sight, and Miguel began to walk towards the prey and mate, as seen in the second picture. In the third and fourth pictures, as Miguel gets closer to the two stimuli, we can see that it is heading for the prey, which is represented by the orange box in the foreground. After contacting the prey with one of its whiskers in the fifth picture, the obstacle-avoidance behavior took over, and the robot backed up, as shown in the sixth picture. This put both the prey and mate in view once again. Since the hunger level had been reset after contacting the prey, Miguel turned toward the mate in the seventh picture, and moved to contact the mate, as shown in the last picture. After the mate was contacted, the predator was placed in view again. Miguel froze and the run was over. (The tethers seen in the pictures are for power, one for the robot and one for the vision board; all computation is performed in real-time on-board Miguel).

Miguel's behavior has advanced incrementally. The execution sequences depicted in Figures 6 and 7 show Miguel's behavior during earlier stages in his development. At the time the execution in Figure 6 was conducted, Miguel was using obstacle-avoidance and prey tracking behaviors. Figure 6 shows Miguel following an orange box, which represents prey, as it is moved around.

Figure 7 shows Miguel running obstacle-avoidance and the move- forward behavior, as well as responding to both prey and predator in the environment. In the first part of the sequence shown in Figure 7, Miguel follows prey, represented by the orange box, as it is moved. Then a predator, represented by a green box, is introduced into the robot's field of view. Miguel stops and remains motionless until the predator is removed. At this point, Miguel resumes his movement toward the prey.

Several other trials have been conducted with Miguel. In some of these, both predator and prey have been placed in view, causing the robot to stop moving, and then left in view long enough that the hunger level becomes greater than the fear level. In this case, the robot starts moving again toward the prey. The same test has been conducted using a predator and mate, with similar results. If there is no stimulus in the environment, then the robot moves forward.

If the robot contacts an obstacle it will back up, turn a little, walk forward a few steps, and then respond to the present view in the environment. Since the robot does not retain any previous locations of stimuli in memory, this can cause the robot to abandon any prey or mate that it had previously been trying to acquire. For instance, if the robot is moving toward prey and contacts an obstacle, then after it backs up, turns, and moves forward, the prey may not be visible anymore. In this case, the robot would abandon its attempt to acquire that prey.

\section{Summary and Conclusions}

We have presented a partial implementation of an ethological model of a praying mantis on a robotic hexapod which incorporates visually guided motivated behaviors such as prey acquisition, mating, and predator avoidance. These were further integrated with obstacle avoidance and chantlitaxia behaviors. Results that were earlier demonstrated in simulation [4] are now shown on a fielded real-time vision-based hexapod. These efforts demonstrate the feasibility of importing models from the biological community into robotics and show that species-specific activities can lead to interesting robotic performance. It is hoped that these results will engender future research within the biological community that will lead to iterative refinement of models such as the one presented here.

\section{Acknowledgments}

This research was supported by National Science Foundation Grant \#IRI-9505864. The authors would like to thank Alfredo Weitzenfeld and Francisco Cervantes-Pérez for their involvement in this project. 

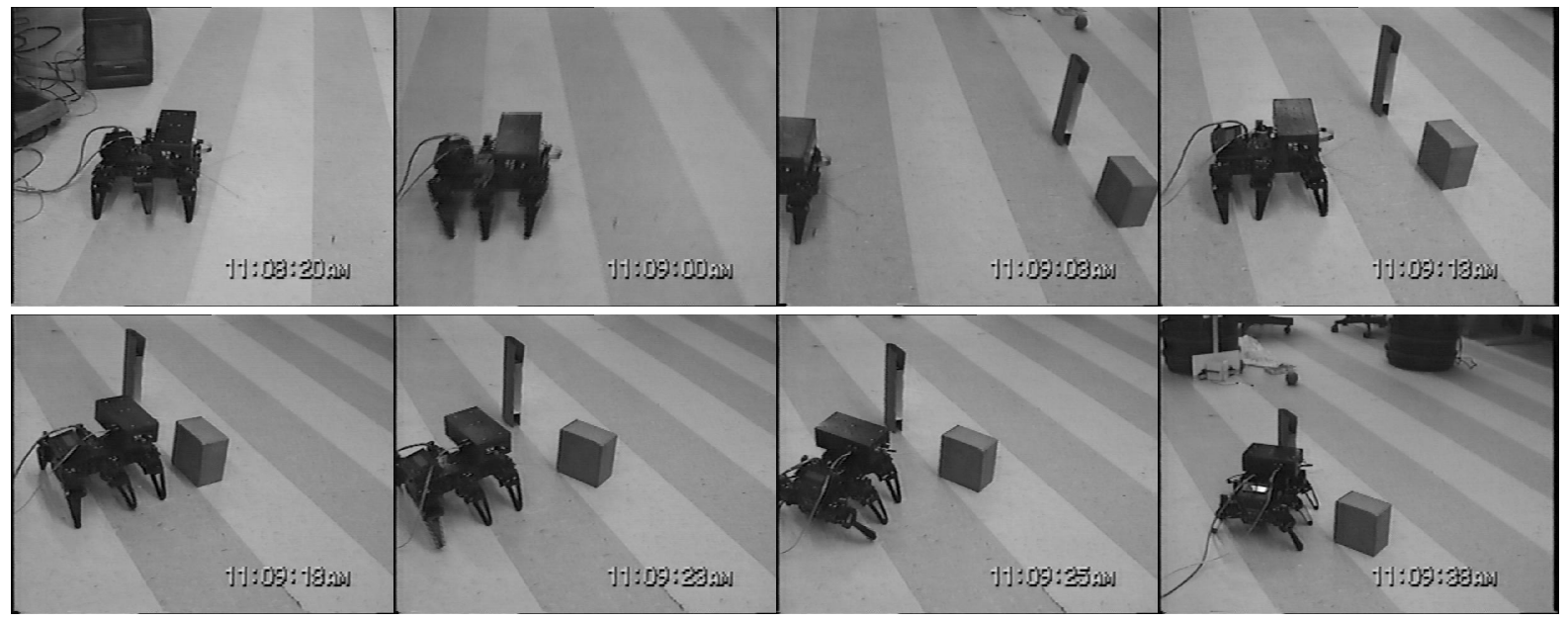

Figure 5: This sequence of pictures depict Miguel's actions during one execution. The sequence proceeds from left to right. At first, Miguel is stationary because there is a box representing a predator just out of the picture to the right. After the predator is removed, Miguel then moves towards the orange box in the foreground, which represents prey. Once it has contacted the prey, it moves toward the purple box in the background, which represents a mate. More details of this execution sequence are given in the text.
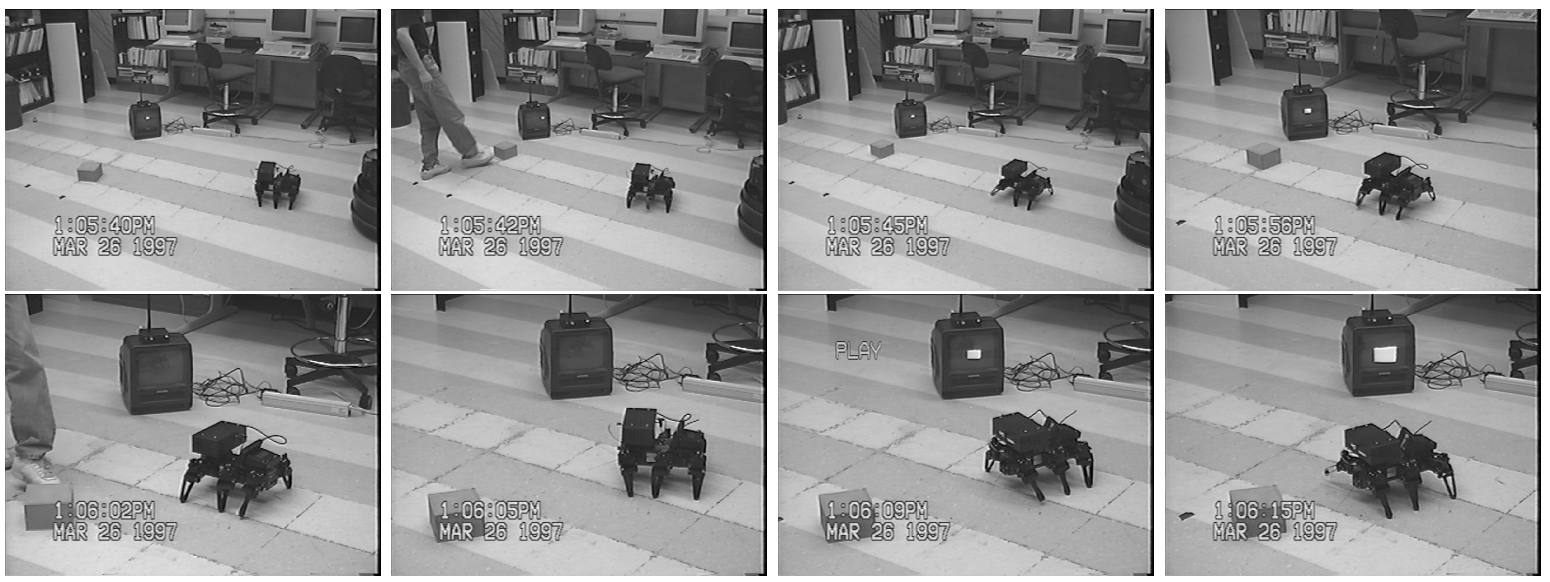

Figure 6: This sequence of pictures shows Miguel following a prey as it is moved around. The sequence proceeds from left to right. The prey is represented by an orange box. The video monitor shows the broadcast results of Miguel's visual processing. Note how in the bottom two photographs the region size increases as Miguel approaches the prey object. 

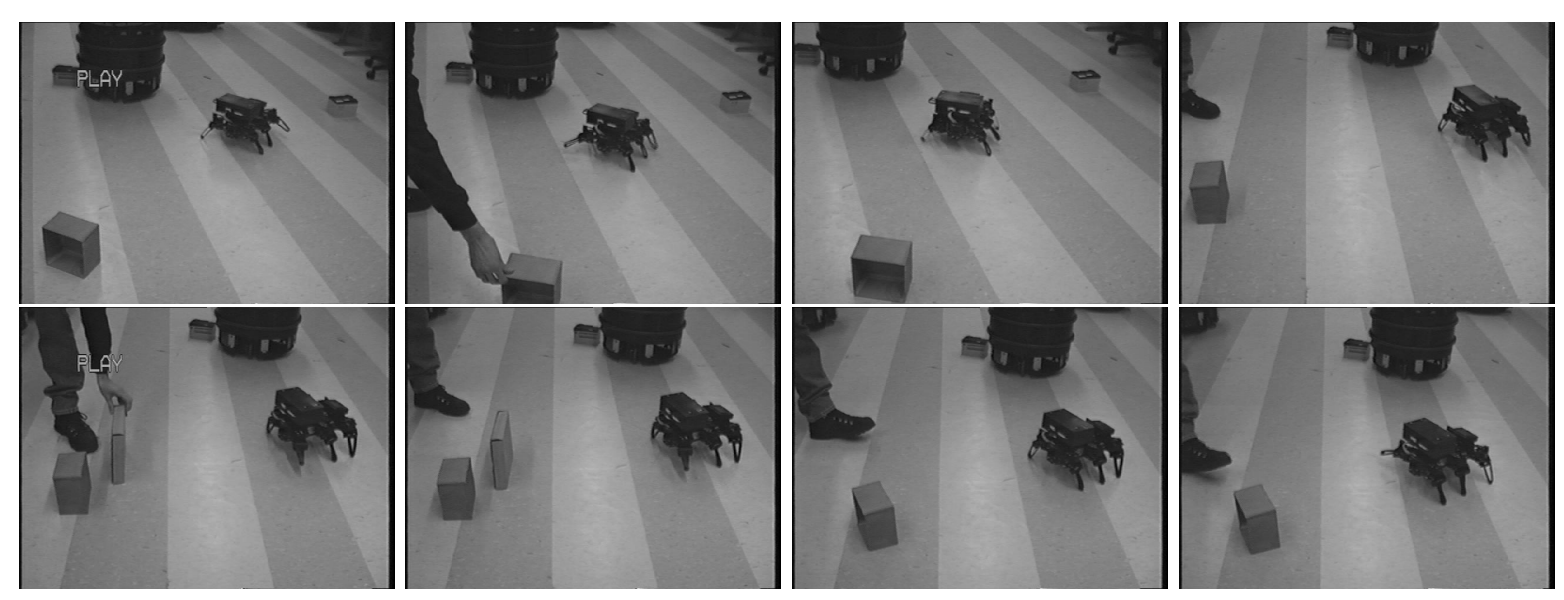

Figure 7: This sequence shows Miguel following prey and reacting to a predator. The sequence proceeds from left to right. In the first four pictures Miguel is moving towards the prey as it is moved around. The prey is represented by an orange box. In the fifth picture, a predator is placed alongside the prey in Miguel's view. A tall green box represents the predator. When Miguel sees the predator, it stops moving, and in the sixth picture, we can see that Miguel has remained motionless. In the last two pictures, the predator has been removed from Miguel's view, and the robot resumes moving toward the prey.

\section{References}

[1] Arbib, M., "Schema Theory", in The Handbook of Brain Theory and Neural Networks, ed. M. Arbib, MIT Press, Cambridge, MA, 1995, pp. 830-834.

[2] Arkin, R.C., "Motor Schema-Based Mobile Robot Navigation", International Journal of Robotics Research, Vol. 8, No. 4, August 1989, pp. 92-112.

[3] Arkin, R.C., "Modeling Neural Function at the Schema Level: Implications and Results for Robotic Control", in Biological Neural Networks in Invertebrate Neuroethology and Robotics, eds. McKenna and Beer, 1993, pp. 383-410.

[4] Arkin, R.C., Cervantes-Pérez, F., and Weitzenfeld, A., "Ecological Robotics: A Schema-Theoretic Approach", to appear Intelligent Robots: Sensing, Modelling and Planning, eds. R.C. Bolles, H. Bunke, and H. Noltemeier, World Scientific, 1998.

[5] Beer, R., Intelligence as Adaptive Behavior: An Experiment in Computational Neuroethology, Academic Press, 1990.

[6] Brooks, R., "A Robust Layered Control System for a Mobile Robot", IEEE Journal of Robotics and Automation, Vol. RA-2, No. 1, 1986 pp. 14-23.

[7] Cervantes-Pérez, F., "Schema Theory as a Common Language to Study Sensori-Motor Coordination" in: Visuomotor coordination: amphibians, comparisons, and robots. Eds. Ewert, J.P., and Arbib, M.A., Plenum Press, 1989, pp. 421-45.

[8] Cervantes-Pérez, F., Franco, A., Velazquez, S., Lara, N., 1993, A Schema Theoretic Approach to Study the
"Chantitlaxia" Behavior in the Praying Mantis, Proc. of the First Workshop on Neural Architectures and Distributed AI: From Schema Assemblages to Neural Networks, USC, October 19-20.

[9] Connell, J., "A Colony Architecture for an Artificial Creature", Technical Report 1151, MIT A.I. Laboratory, August 1989.

[10] Langton, C., (ed.) Artificial Life: An Overview, MIT Press, Cambridge, MA, 1995.

[11] Grand, S., Cliff, D., Malhotra, A., "Creatures: Artificial Life Autonomous Software Agents for Home Entertainment", Proc. Autonomous Agents '97, Marina Del Rey, CA, Feb., 1997.

[12] Lyons, D. and Arbib, M., "A Formal Model of Computation for Sensory-based Robotics", IEEE Trans. on Robotics and Auto., Vol. 6, No. 3, June 1989, pp. 280-293.

[13] Maes, P., "The Dynamics of Action Selection" Proc. Eleventh Intern. Joint Conf. on Artificial Intelligence (IJCAI-89), Detroit, MI, pp. 991-997, 1989.

[14] Mataric, M., "Navigating with a Rat Brain: A Neurobiologically-Inspired Model for Robot Spatial Representation", Proc. 1st Int. Conf. on Simulation of Adaptive Behavior, 1990, pp. 169-75. 